\title{
Introduction to the Special Section of the 2019 APMF, the Asia Pacific Measurement Forum on Mechanical Quantities
}

\author{
Koji Ogushi ${ }^{1}$, Momoko Kojima ${ }^{1}$ \\ ${ }^{1}$ National Metrology Institute of Japan, National Institute of Advanced Industrial Science and Technology, Tsukuba Central 3, 1-1-1 \\ Umezono, Tsukuba, 305-8563 Ibaraki, Japan
}

Section: EDITORIAL

Citation: Koji Ogushi, Momoko Kojima, Introduction to the Special Section of the 2019 APMF, the Asia Pacific Measurement Forum on Mechanical Quantities, Acta IMEKO, vol. 10, no. 1, article 2, March 2021, identifier: IMEKO-ACTA-10 (2021)-01-02

Editor: Francesco Lamonaca, University of Calabria, Italy

Received March 17, 2021; In final form March 17, 2021; Published March 2021

Copyright: This is an open-access article distributed under the terms of the Creative Commons Attribution 3.0 License, which permits unrestricted use, distribution, and reproduction in any medium, provided the original author and source are credited.

Corresponding authors: Koji Ogushi, e-mail: kji.ogushi@aist.go.jp

Momoko Kojima, e-mail: m.kojima@aist.go.jp

Dear Readers,

Measurement technology on mass, force, torque constitutes an integral part of intellectual infrastructure for a diverse range of human activities such as quality and safety assurance of industrial products, fair trade, energy-saving, and environmental protection. The Asia Pacific Symposium on Measurement of Mass, Force and Torque (APMF), since its initiation in 1992, has been offering participants the opportunity of exchanging the latest information on $\mathrm{R} \& \mathrm{D}$ in these fields. It has been growing steadily as a not-to-miss event for metrologists, researchers, and engineers, especially those actively working in the Asia-Pacific region.

The name "Asia Pacific Measurement Forum on Mechanical Quantities (APMF)" has been considered and changed from "Asia Pacific Symposium on Measurement of Mass Force and Torque (APMF)" in 2017. Besides, APMF activities' scope has been extended into different mechanical quantities such as density, hardness, pressure, vacuum, and others.

The APMF 2019 has been held in Niigata, Japan, from $17^{\text {th }}$ to $21^{\text {st }}$ November 2019. It has been sponsored by the Society of Instrument and Control Engineers (SICE), co-sponsored by the International Measurement Confederation (IMEKO) and Niigata University, and organized by the National Metrology Institute of Japan (NMIJ), a division of the National Institute of Advanced Industrial Science and Technology (AIST). The successful forum was contributed by more than 140 participants from 14 countries and economies, presenting 71 scientific papers. In this Special Issue, you can find four articles selected by the International Program Committee of the APMF, which is considered worthy of publishing in the Acta IMEKO journal after peer-reviewing.

The papers included in the special section show recent progress of the research on the measurements in the fields of mass, pressure, and flow in the Asia-Pacific region. We briefly introduce those papers as follows.

In the field of mass metrology, one paper was selected. Yu$\mathrm{Hsin} \mathrm{Wu}$ and her colleagues presented the combined X-ray fluorescence (XRF) / X-ray photoelectron spectroscopy (XPS) surface analysis system for quantitative surface layer analysis of $\mathrm{Si}$ spheres in order to realize the new $\mathrm{kg}$ definition by X-ray crystal density (XRCD) method in CMS/ITRI. PTB cooperated with CMS by transmitting the information and technology of the XRCD method.

In the field of pressure metrology, two papers are included in the issue. Ahmed S. Hashad and PTB team reported the evaluation of PTB Force-Balanced Piston Gauge (FPG), which is a non-rotating piston gauge. They have compared FPG with three different PTB pressure standards ranging from $3 \mathrm{~Pa}$ to 15 $\mathrm{kPa}$ and confirmed the theoretically obtained effective area. The other is the topic about improvement of YOKOGAWA's silicon resonant pressure transducer reported by Hideaki Yamashita. The characteristics of the pressure sensors are refined excellently based on the calibration results from NMIJ and YOKOGAWA, which aimed to be used as a transfer standard in future key comparisons. In the flow field, Masanao Kaneko has numerically investigated the effect of a single groove on the flow behaviour and loss generation in a linear compressor cascade. Analysis was performed by changing the tip clearance, which will be beneficial for the improvement of the compressor aerodynamic performance in the future.

We are deeply grateful to all contributors, editors, authors, and reviewers who make this issue possible and hope you will enjoy reading this special section.

Koji Ogushi, Momoko Kojima

Guest editors 